\title{
LOS MERCADOS ANDINOS Y SUS REQUERIMIENTOS DE MAQUINARIA AGRÍCOLA
}

\section{THE ANDEAN MARKETS AND THEIR REQUIREMENTS FOR AGRICULTURAL MACHINERY}

\author{
Melton Tapia Zurita ${ }^{1}$ \\ Edison Tapia Zurita ${ }^{2}$
}

1. Ing. Mecánico, Máster en Mecatrónica por la Universidad Politécnica de Cataluña, Docente, Coordinador del área Mecatrónica del Departamento de Ciencias de la Energía y Mecánica de la Universidad de Fuerzas Armadas - ESPE, provincia de Pichincha, Ecuador. E-mail: metapia@espe.edu.ec

2. Ing. Mecánico, Máster en Materiales, Diseño y Automatización por la Escuela Politécnica Nacional de Quito, jefe de proyectos SEDEMI (Soluciones en Estructuras Metálicas), provincia de Pichincha, Ecuador. E-mail: gustavo.zur@hotmail.com

\section{Citación sugerida:}

Tapia Zurita, M. y Tapia Zurita, E. (2017). Los mercados andinos y sus requerimientos de maquinaria agrícola. 3C Empresa, investigación y pensamiento crítico, 6(3), 47-62. DOI: <http://dx.doi.org/10.17993/3cemp.2017.060331.47-62/>. 


\section{RESUMEN}

El trabajo muestra los resultados del estudio desarrollado en la Región Andina ecuatoriana para determinar los requerimientos de maquinaria agrícola en los procesos productivos de mayor relevancia y generar soluciones basadas en las necesidades específicas de los productores, considerando además su situación socioeconómica. Con esta información se determinan los procesos de mayor interés en el desarrollo de equipamiento y las propuestas de solución, tomando en cuenta que en la región predominan las pequeñas y medianas unidades familiares productivas agropecuarias. El aporte de este trabajo es dar a conocer las necesidades de equipamiento en los procesos específicos de producción, la factibilidad de desarrollar este equipamiento y el interés por adquirir el mismo por parte de los productores.

\section{ABSTRACT}

The work shows the results of the study carried out in the Ecuadorian Andean Region to determine the requirements of agricultural machinery in the most relevant productive processes and to generate solutions based on specific needs of the producers, considering in addition their socioeconomic situation. With this information, the most important processes in the development of equipment and the proposed solutions are determined, taking into account that the region is dominated by small and medium productive agricultural family units. The contribution of this work is to make known the needs of equipment in specific processes of production, the feasibility of developing this equipment and the interest to acquire it by producers.

\section{PALABRAS CLAVE}

Región Andina, requerimientos, maquinaria agrícola, necesidades, especificaciones.

\section{KEY WORDS}

Andean Region, requirements, agricultural machinery, needs, specifications. 


\section{INTRODUCCION}

"El sector de maquinaria agrícola es una industria metalmecánica de bienes de capital que abarca gran variedad de productos que contribuyen a mejorar y optimizar las principales actividades agrícolas y ganaderas" (Albornoz, 2010). Para lograr el incremento de la competitividad en la Región Andina se debe reforzar la innovación, agregar valor a los productos y hacer que los mismos se diferencien partiendo de puntos de vista culturales, ambientales y territoriales, así como es fundamental que se vincule la agricultura familiar a los mercados (CEPAL, FAO, IICA, 2015). Para ello, se requiere un cambio en las técnicas de producción y obligatoriamente la mecanización adaptada a las necesidades de los productores y/o la automatización de los procesos.

En América Latina y el Caribe se estima que existen 60 milllones de personas que trabajan en 17 millones de unidades productivas que corresponden a unidades de agricultura familiar. En algunos países de la región,esta forma de trabajo representa hasta el $90 \%$ de las unidades productivas (CEPAL, FAO, IICA, 2013).

La falta de tecnificación debida al desconocimiento y los escasos recursos económicos de los productores hace que exista un pobre rendimiento de las tierras (De los Ríos, 2008). La oferta de equipamiento para tecnificar los procesos de producción de cultivos andinos es prácticamente nula. Ello es debido a que al ser un mercado que si bien es muy grande está muy limitado de recursos, y las grandes corporaciones fabricantes de maquinaria no atienden al sector ni investigan acerca de sus productos. Así, se deja este campo para que sea atendido por pequeños fabricantes de equipamiento que trabajan con los pocos productores interesados en mejorar su forma de trabajo, haciéndolo de manera inadecuada y generando soluciones no eficientes y con costos muchas veces inaccesibles a ellos.

Gleason (2006), en un estudio realizado en México para conocer las necesidades del mercado de maquinaria agrícola, menciona que "el pequeño agricultor tiene urgencia de maquinaria que satisfaga los siguientes requisitos:

1. Maquinaria barata al alcance de sus posibilidades.

2. Maquinaria fuerte pero económica que se puede transportar con facilidad.

3. Maquinaria sencilla y económica en su manejo.

4. Maquinaria que proporcione rendimientos adecuados a la producción agrícola.

5. Maquinaria cuyas refacciones sean baratas.

6. Maquinarias que se acondicionen especialmente a las diversas regiones del país teniendo en cuenta la naturaleza del terreno, los métodos de cultivo, etc.

7. Maquinaria cuyas reparaciones sean fáciles y estén al alcance del agricultor" (Gleason, 2006, p. 148). 
Estos requerimientos son similares a los solicitados por los productores en el estudio realizado y expuesto en este artículo.

Las transformaciones agrícolas, acompañadas de aportes y apoyo de sociedades tecnificadas logran como resultado el incremento de divisas y el desarrollo industrial. Ejemplo de este fenómeno es el caso taiwanés, en el cual, los cambios en sus formas agrícolas a pequeña escala y la ayuda norteamericana fueron las bases del gran crecimiento industrial, siendo este uno de los mejores ejemplos de desarrollo basados en la pequeña explotación (Pérez, 1991); de ahí la importancia del trabajo y oferta de equipamiento adecuado a las necesidades del pequeño productor.

Los países andinos, excepto Brasil y México, son todos importadores de maquinaria agrícola (Albornoz, 2010), pero esta provisión solamente se enfoca a los grandes productores.

El incremento de la población en los países latinoamericanos ha hecho que exista una mayor demanda de alimentos, reduciéndose a la vez las tierras productivas, lo cual hace imprescindible la mejora de eficiencia en la producción para poder abastecer de los productos tradicionales (Albornoz, 2010). Sumado a esto, existe el problema de la migración campesina que afecta no solo a la población urbana sino a la rural, pues las tierras dejan de ser productivas por falta de mano de obra (Martinez, 2005).

La Organización de las Naciones Unidas para la Alimentación y la Agricultura (FAO) en una publicación realizada en el año 2014 sugiere a la industria mundial proporcionar mayor apoyo a los pequeños agricultores realizando diseños que se adapten a sus necesidades (CEPAL, FAO, IICA, 2014). Kienzle, J., Ashburner, J. y Sims, B., (2013) mencionan en su estudio que esto ayudaría a los productores a realizar sus procesos con mayor efectividad $y$ rendimiento y los impulsaría a salir de la pobreza.

El desarrollo y comercialización de equipamiento, adecuado y adaptado a las realidades técnicas y socioeconómicas de los pequeños y medianos productores garantizará la seguridad alimentaria, crecimiento económico, mitigación de pobreza y protección del medio ambiente (Kienzle, J., Ashburner, J. y Sims, B., 2013).

La región produce variedad de cultivos, entre ellos, tubérculos, raíces, granos, leguminosas, frutales y cultivos andinizados (Tapia, M., Fries, A., 2007). Debido a esta gran variedad, el trabajo se concentra en el mercado de maquinaria aplicado a granos y cultivos andinizados.

En América Latina existen políticas públicas relacionadas al apoyo y financiamiento para la producción, enfocadas básicamente a pequeños y medianos productores. Estas se han desarrollado debido a la importancia que este sector ha adquirido en las últimas décadas (Sabourin, E., Samper, M., Le Coq, J., Massardier, G., Sotomayor, O., 2014), condición que podría contribuir a que exista demanda de la maquinaria aplicada al desarrollo de este sector productivo. 


\section{ANTECEDENTES}

Este trabajo de investigación comenzó en el año 2012 con el proyecto denominado NewAgri-Now, "Análisis de los procesos agroalimentarios para el establecimiento de las nuevas especificaciones de equipos agrícolas para el procesado de alimentos en base a los contextos socioeconómicos y medio ambientales", que fue financiado por la Asociación Española de Cooperación Internacional para el Desarrollo (AECID) y ejecutado por las Universidades Politécnica de Cataluña de España (UPC) y Politécnica del Ejército de Ecuador (ESPE). La figura 1 muestra la noticia relacionada al proyecto que se encuentra publicada en la página web de la UPC (CDEI-UPC, 2012).

Inicio · Comunicación · Noticias

\section{Noticies}

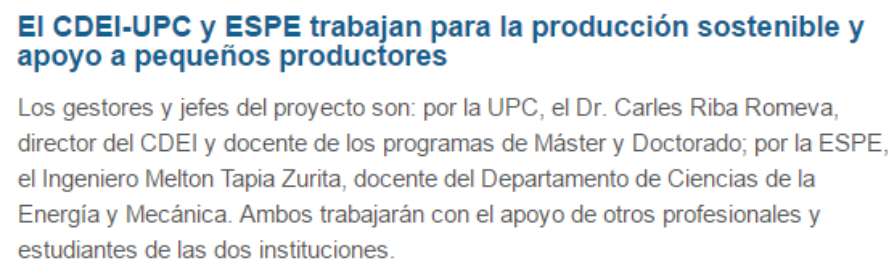

El objetivo del proyecto es presentar un modelo de maquinaria, respetuoso con el medio ambiente y de bajo costo, adaptado a la realidad socio económica ecuatoriana. La meta de esta investigación es apoyar a la producción agrícola en los sectores más necesitados. Se espera como resultado fundamental, obtener un modelo industrial de maquinaria que pueda ser patentable.

Figura 1. Noticia relacionada al desarrollo del proyecto inicial de trabajo.

Fuente: CDEI - UPC.

Como parte de las actividades del proyecto se realizó el estudio de los requerimientos de los productores en varias regiones del Ecuador. Con esta información se definieron los productos, sectores y procesos en los que se centraría el trabajo. Como resultado de esta cooperación se diseñó y fabricó un equipo deshojador de maíz para la variedad seco suave de altura que fue donado al Instituto Nacional de Investigaciones Agropecuarias (INIAP). Posteriormente, en la Universidad de fuerzas Armadas - ESPE hasta la actualidad, se ha continuado con los estudios para desarrollar nuevos prototipos de apoyo al agro, enfocados básicamente a granos andinos y productos andinizados. 


\section{METODOLOGÍA}

Debido a la gran variedad de productos y procesos, para definir los equipos que requieren estudio y desarrollo, se realizaron análisis mediante matrices de ponderación, utilizando la metodología propuesta por Carles Riba en su libro de diseño concurrente (Riba, 2002). Los criterios ponderados así como las propuestas de equipamiento fueron establecidos en base a las necesidades de los agricultores y de factores analizados por el equipo de desarrollo.

La figura 2 muestra la matriz de ponderación de criterios en donde la columna final de la tabla muestra el valor fraccional del peso de cada criterio.

\begin{tabular}{|c|c|c|c|c|c|c|c|c|c|c|c|c|c|c|}
\hline & $\begin{array}{c}\text { No. } \\
\text { USUARIOS } \\
\text { UPAs (A) } \\
\end{array}$ & $\begin{array}{c}\text { ENCUESTA } \\
\text { DE } \\
\text { USUARIOS } \\
(\mathrm{B})\end{array}$ & $\begin{array}{c}\text { VIABILIDA } \\
\text { D. } \\
\text { FABRICAC. } \\
\text { (C) }\end{array}$ & $\begin{array}{l}\text { VIABILIDA } \\
\text { D. DISEÑO } \\
\end{array}$ & $\begin{array}{l}\text { PROD. } \\
\text { SIMILARES } \\
\text { USADOS } \\
\text { ECUADOR }\end{array}$ & $\begin{array}{c}\text { TIEMPO } \\
\text { DE } \\
\text { DESARROL } \\
\text { LO } \\
\end{array}$ & \begin{tabular}{|c|} 
PROD. \\
SIMILARES \\
MUNDO \\
\end{tabular} & $\begin{array}{c}\text { COSTE DE } \\
\text { MAQUIN } \\
\text { ARIA } \\
\end{array}$ & $\begin{array}{c}\text { DISPON } \\
\text { IBILIDA } \\
\text { D }\end{array}$ & $\begin{array}{c}\text { REQU. } \\
\text { ENERGÉ } \\
\text { TICO DE } \\
\text { USO } \\
\end{array}$ & $\begin{array}{c}\text { IMPACT } \\
0 \\
\text { ENERGÉ } \\
\text { TICO EN } \\
\text { EL } \\
\text { PROCES } \\
0 \\
\end{array}$ & $\begin{array}{c}\text { OTROS } \\
\text { IMPACT } \\
\text { OS } \\
\text { AMBIEN } \\
\text { TALES } \\
\end{array}$ & $\Sigma+1$ & Pondera. \\
\hline No. USUARIOS UPAs (A) & & 2,5 & 0 & 0 & 2,5 & 2,5 & 2,5 & 0 & 2,5 & 2,5 & 2,5 & 2,5 & 21 & 0,06 \\
\hline ENCUESTA DE USUARIOS (B) & 2,5 & & 0 & 0 & 2,5 & 2,5 & 2,5 & 0 & 0 & 0 & 0 & 0 & 11 & 0,03 \\
\hline VIABILIDAD. FABRICAC. (C) & 5 & 5 & & 2,5 & 5 & 5 & 5 & 0 & 5 & 2,5 & 2,5 & 0 & 38,5 & 0,11 \\
\hline VIABILIDAD. DISENNO & 5 & 5 & 2,5 & & 5 & 5 & 5 & 0 & 5 & 2,5 & 2,5 & 0 & 38,5 & 0,11 \\
\hline PROD. SIMILARES ECUADOR & 2,5 & 2,5 & 0 & 0 & & 2,5 & 2,5 & 0 & 0 & 0 & 0 & 2,5 & 13,5 & 0,04 \\
\hline TIEMPO DE DESARROLLO & 2,5 & 2,5 & 0 & 0 & 2,5 & & 2,5 & 0 & 0 & 0 & 0 & 0 & 11 & 0,03 \\
\hline PROD. SIMILARES MUNDO & 2,5 & 2,5 & 0 & 0 & 2,5 & 2,5 & & 0 & 0 & 0 & 0 & 0 & 11 & 0,03 \\
\hline COSTE DE MAQUINARIA & 5 & 5 & 5 & 5 & 5 & 5 & 5 & & 5 & 2,5 & 2,5 & 2,5 & 48,5 & 0,14 \\
\hline DISPONIBILIDAD & 2,5 & 5 & 0 & 0 & 5 & 5 & 5 & 0 & & 0 & 2,5 & 2,5 & 28,5 & 0,08 \\
\hline REQU. ENERGÉTICO DE USO & 2,5 & 5 & 2,5 & 2,5 & 5 & 5 & 5 & 2,5 & 5 & & 2,5 & 0 & 38,5 & 0,11 \\
\hline $\begin{array}{l}\text { IMPACTO ENERGÉTICO EN EL } \\
\text { PROCESO }\end{array}$ & 2,5 & 5 & 2,5 & 2,5 & 5 & 5 & 5 & 2,5 & 2,5 & 2,5 & & 0 & 36 & 0,11 \\
\hline OTROS IMPACTOS AMBIENTALES & 2,5 & 5 & 5 & 5 & 2,5 & 5 & 5 & 2,5 & 2,5 & 5 & 5 & & 46 & 0,13 \\
\hline & & & & & & & & & & & & Suma & 342 & 1,00 \\
\hline
\end{tabular}

Figura 2. Matriz de ponderación de criterios.

Fuente: elaboración propia.

Se evaluaron posteriormente cada una de las opciones de desarrollo de equipamiento respecto a cada uno de los criterios. Finalmente, se obtuvo la matriz de resultados en la que se definieron los equipos que potencialmente debían desarrollarse.

La figura 3 muestra la matriz de resultados con la ponderación final de los equipos que requieren desarrollo.

\begin{tabular}{|c|c|c|c|c|c|c|c|c|c|c|c|c|c|c|}
\hline & 1 & 2 & 3 & 4 & 5 & 6 & 7 & 8 & 9 & 10 & 11 & 12 & suma & Prioridad \\
\hline Trillador & 0,0033 & 0,0046 & 0,0226 & 0,0206 & 0,0056 & 0,0046 & 0,0046 & 0,0203 & 0,0119 & 0,0161 & 0,0150 & 0,0192 & 0,1484 & 1 \\
\hline tamizador & 0,0005 & 0,0003 & 0,0151 & 0,0216 & 0,0056 & 0,0046 & 0,0046 & 0,0203 & 0,0119 & 0,0161 & 0,0150 & 0,0192 & 0,1348 & 7 \\
\hline sembrador/abonadora maiz & 0,0143 & 0,0039 & 0,0196 & 0,0116 & 0,0048 & 0,0046 & 0,0046 & 0,0203 & 0,0119 & 0,0136 & 0,0179 & 0,0192 & 0,1460 & 3 \\
\hline deshierbador/removedor maíz & 0,0143 & 0,0082 & 0,0106 & 0,0090 & 0,0065 & 0,0046 & 0,0046 & 0,0203 & 0,0119 & 0,0111 & 0,0179 & 0,0192 & 0,1380 & 6 \\
\hline Deshojador/desgranador maíz & 0,0143 & 0,0082 & 0,0095 & 0,0106 & 0,0056 & 0,0046 & 0,0046 & 0,0203 & 0,0119 & 0,0136 & 0,0179 & 0,0192 & 0,1402 & 5 \\
\hline desgranador & 0,0088 & 0,0024 & 0,0176 & 0,0231 & 0,0056 & 0,0046 & 0,0046 & 0,0203 & 0,0119 & 0,0236 & 0,0056 & 0,0192 & 0,1474 & 2 \\
\hline deshojador & 0,0060 & 0,0046 & 0,0176 & 0,0161 & 0,0056 & 0,0046 & 0,0046 & 0,0203 & 0,0119 & 0,0186 & 0,0160 & 0,0192 & 0,1451 & 4 \\
\hline
\end{tabular}

Figura 3. Matriz de conclusiones en base a criterios.

Fuente: elaboración propia. 
Las ponderaciones de cada una de las opciones de desarrollo fueron similares, por ello se decidió trabajar en el diseño de todos los equipos propuestos relacionados a las etapas de siembra y cosecha de los productos.

Para la determinación de especificaciones, objetivo de los prototipos, se realizaron 50 encuestas a productores de la sierra centro, 50 de la norte y 50 de la sierra sur del Ecuador. El número de encuestas realizadas es el recomendado como un número máximo de encuestas para la obtención de información de necesidades de desarrollo de productos (Griffin, A. y Hauser, J., 1993).

Abbie Griffin y John Hauser en su artículo titulado "The voice of the customer" (la voz del consumidor), indican que hasta el $100 \%$ de necesidades para el desarrollo de productos se pueden obtener investigando a 30 clientes; llegan a esta conclusión utilizando una distribución beta - binomial en la que el valor esperado de $E_{n}$ de la probabilidad de observar una necesidad de $\mathrm{n}$ clientes o usuarios potenciales es:

$E n=1-((\Gamma(n+\beta) \Gamma(\alpha+\beta)) /(\Gamma(n+\alpha+\beta) \Gamma(\beta)))$

Con ello, utilizando la distribución beta binomial y comparando con las observaciones realizadas, se tiene lo expuesto en la figura 4.

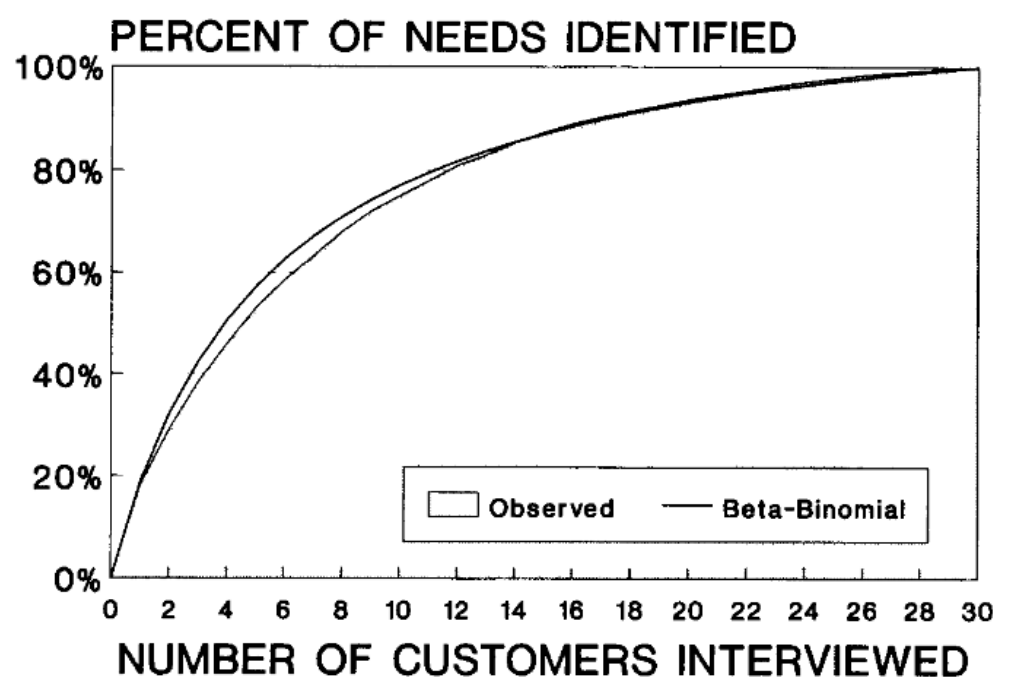

Figura 4. Porcentaje de necesidades identificadas para n clientes. Fuente: Griffin y Hauser, the voice of the customer (1993).

La figura 5 muestra el trabajo de recolección de información de necesidades de equipamiento para el establecimiento de especificaciones objetivo de los equipos. 


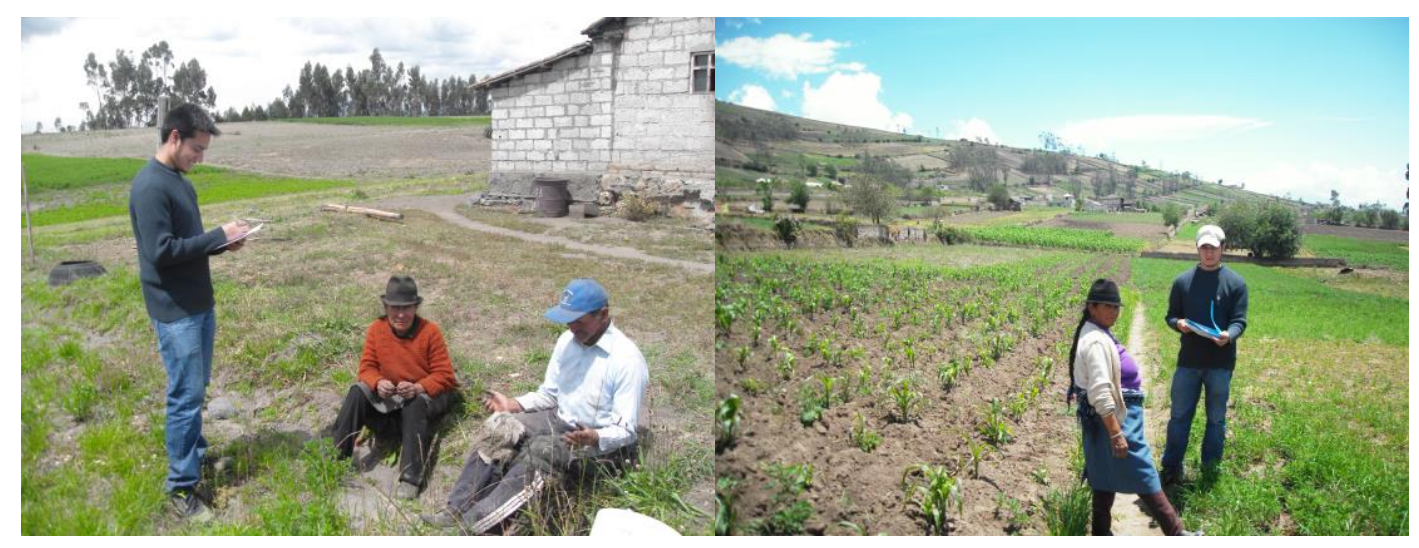

Figura 5. Recolección de información mediante encuestas personales a productores de la sierra centro del Ecuador.

Fuente: autores (2014).

Una vez obtenidos los resultados de las encuestas, se interpretó la información de manera técnica para proceder a especificar el equipamiento.

Posterior a la determinación de especificaciones, del análisis de soluciones existentes y de proyecciones de costos de desarrollo, se fabricaron prototipos funcionales que fueron probados con la finalidad de redefinir las especificaciones de desarrollo. Los prototipos finales se muestran en el apartado de resultados.

En la figura 6 se muestran imágenes de uno de los trabajos de difusión y pruebas.

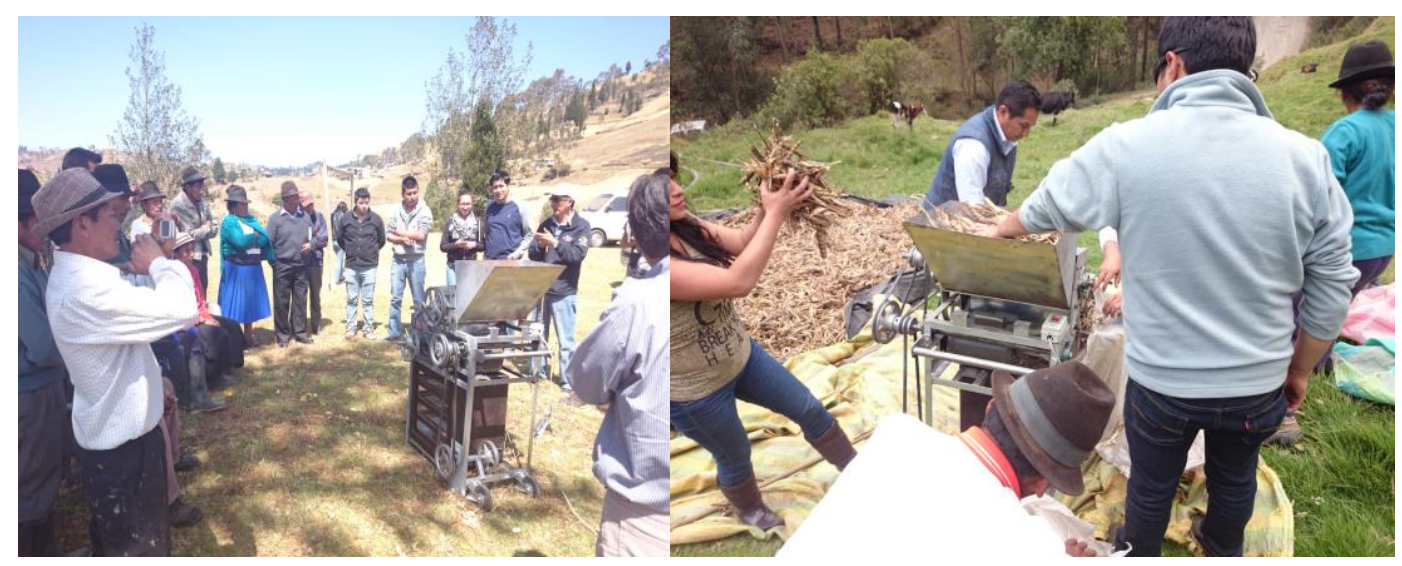

Figura 6. Prototipo trillador difundido y probado con los potenciales usuarios de las comunidades de Bramadero Grande y Bramadero Chico, provincia de Bolívar, Ecuador.

Fuente: autores (2016). 


\section{RESULTADOS}

En esta sección se muestran los resultados de las necesidades de equipamiento para el trabajo así como información de los prototipos desarrollados para solucionar estos requerimientos.

La figura 7 muestra el porcentaje de productores que indicaron requerir maquinaria y los productos en los cuales existe mayor necesidad.

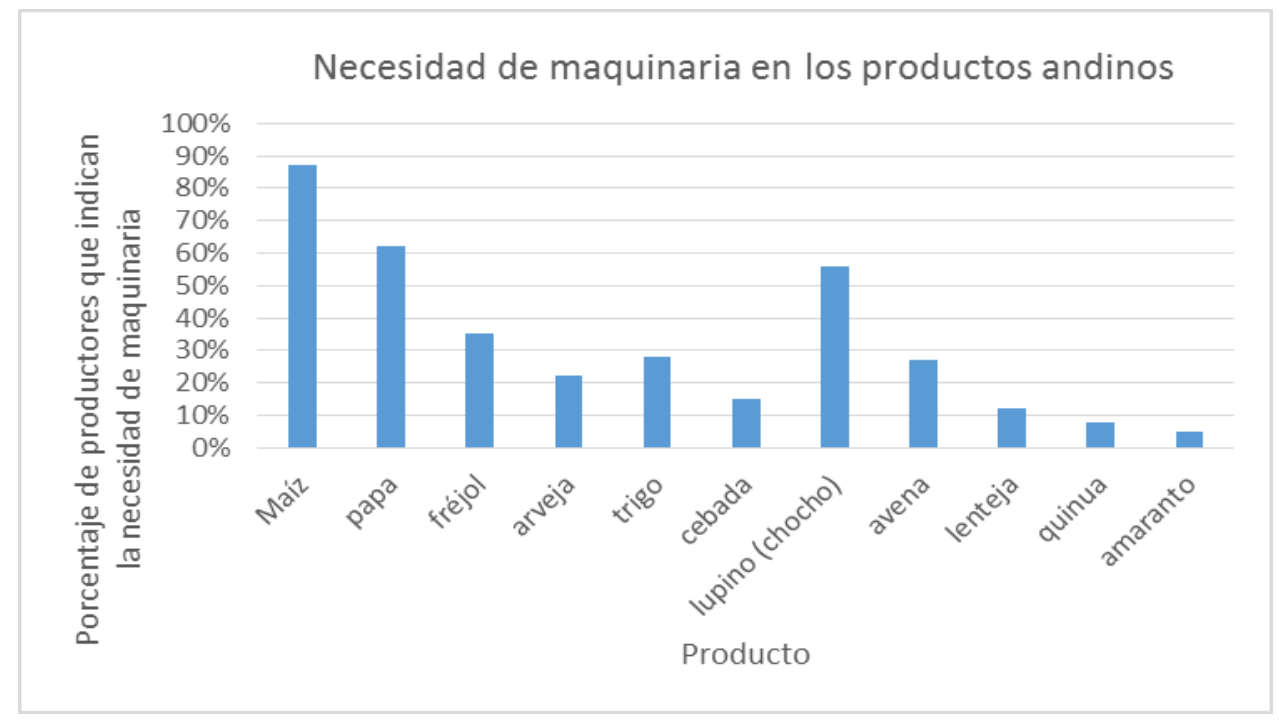

Figura 7. Porcentaje de productores que requieren equipamiento en productos andinos. Fuente: Elaboración propia.

El mayor requerimiento es para producción de maíz, seguido por el de papa. Esta información concuerda con los resultados del censo nacional completo del año 2016 realizado por el Ministerio de Agricultura, Acuacultura y Pesca del Ecuador (Sinagap, 2016) en el que se muestra que en el país fueron cultivadas 105.400 ha. de maíz suave entre seco y duro, seguidos por los cultivos de papa que fueron de $47.494 \mathrm{Ha}$. sembradas. Después de estos productos, los de mayor siembra son fréjol con $19.438 \mathrm{Ha}$. y arveja con $5.919 \mathrm{Ha}$. Otros productos como el lupinus (popularmente llamado Chocho), es un producto que los agricultores siembran, pero en menor proporción debido a su laboriosidad en la cosecha.

De la muestra consultada, en relación a la utilización de equipamiento agrícola (a excepción de lo relacionado a preparación del suelo) en alguna etapa del proceso de producción se determinó que apenas un $2 \%$ de los encuestados la había utilizado en algún momento y en alguna etapa.

En relación a conocer de la existencia de equipamiento para procesos específicos de trabajo, un $28 \%$ conoce que existe maquinaria, el resto no sabe de la misma.

Un 100\% de la muestra desconoce que la falta de mecanización provoca pérdidas en las cosechas, este factor podría incidir en el interés por su adquisición. 
La tabla 1 muestra las etapas representativas (que generan mayor esfuerzo y gasto al productor) de trabajo para los productos más representativos según el estudio.

Tabla 1. Productos andinos con mayor demanda de equipamiento y sus procesos de producción.

\begin{tabular}{|c|c|}
\hline PRODUCTO & PROCESOS DE PRODUCCIÓN \\
\hline \multirow{7}{*}{ MAÍZ } & SIEMBRA \\
\hline & FERTILIZACIÓN \\
\hline & DESHIERBA \\
\hline & RECOLECCIÓN \\
\hline & DESHOJE \\
\hline & DESGRANE \\
\hline & CLASIFICACIÓN POR TAMAÑOS \\
\hline \multirow{6}{*}{ PAPA } & SIEMBRA \\
\hline & DESHIERBA \\
\hline & FERTILIZACIÓN \\
\hline & FUMIGACIÓN \\
\hline & COSECHA \\
\hline & CLASIFICACIÓN POR TAMAÑOS \\
\hline \multirow{5}{*}{ LUPINUS (CHOCHO) } & SIEMBRA \\
\hline & FERTILIZACIÓN \\
\hline & RECOLECCCIÓN \\
\hline & TRILLA \\
\hline & LIMPIEZA \\
\hline \multirow{5}{*}{ FRÉJOL } & SIEMBRA \\
\hline & FERTILIZACIÓN \\
\hline & RECOLECCIÓN \\
\hline & TRILLA \\
\hline & LIMPIEZA \\
\hline
\end{tabular}

Fuente: elaboración propia.

La figura 8 muestra las necesidades de equipamiento en el proceso de producción de maíz suave.

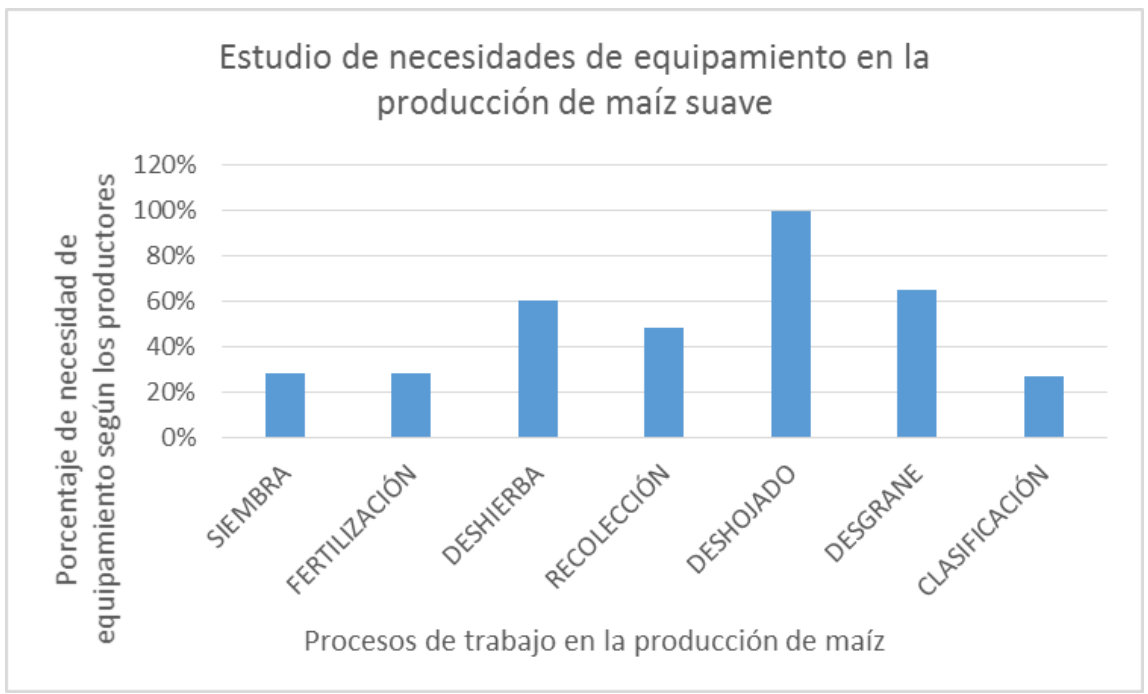

Figura 8. Necesidades de equipamiento en el proceso de producción de maíz suave.

Fuente: elaboración propia. 
La producción de maíz, según las necesidades actuales de los productores, requiere de equipamiento básicamente en el proceso de retiro de la hoja; el proceso de desgrane y clasificación tiene menor importancia en cuanto a tecnificación pero de igual modo lo consideran un proceso que requiere apoyo.

La figura 9 expone los resultados de las necesidades de equipamiento en la producción de papa.

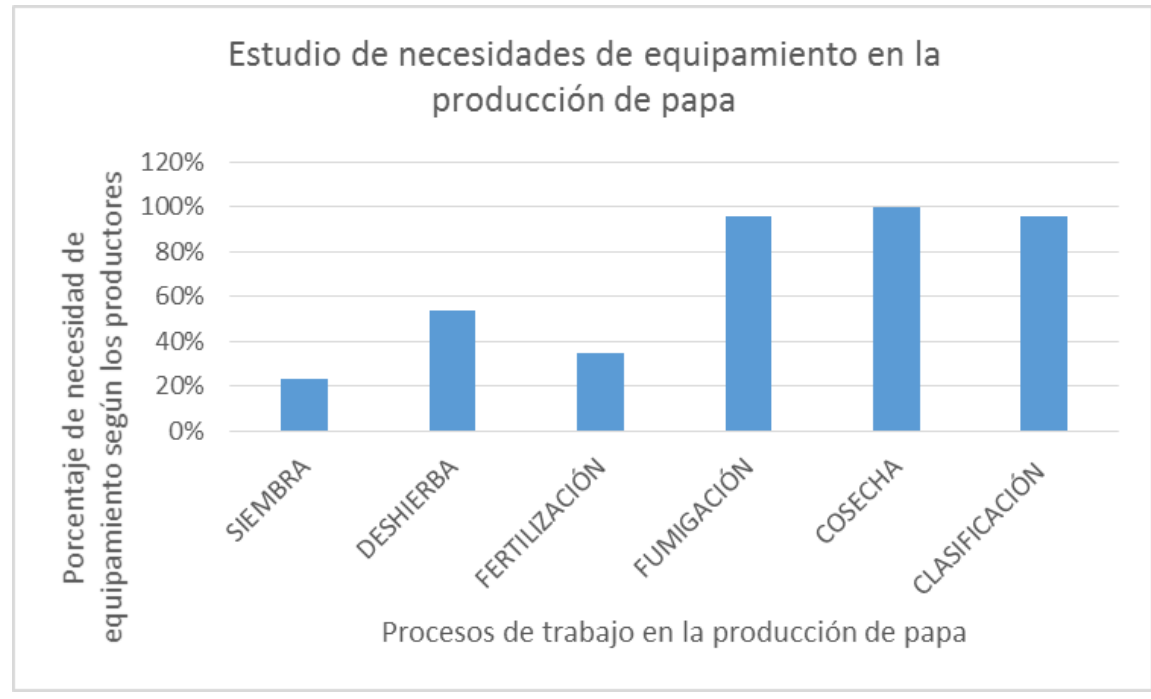

Figura 9. Necesidades de equipamiento en el proceso de producción de papa. Fuente: elaboración propia.

En la producción de papa, el mayor requerimiento se lo encuentra en la etapa de cosecha.

La figura 10 muestra las necesidades de equipamiento en la producción de lupinus.

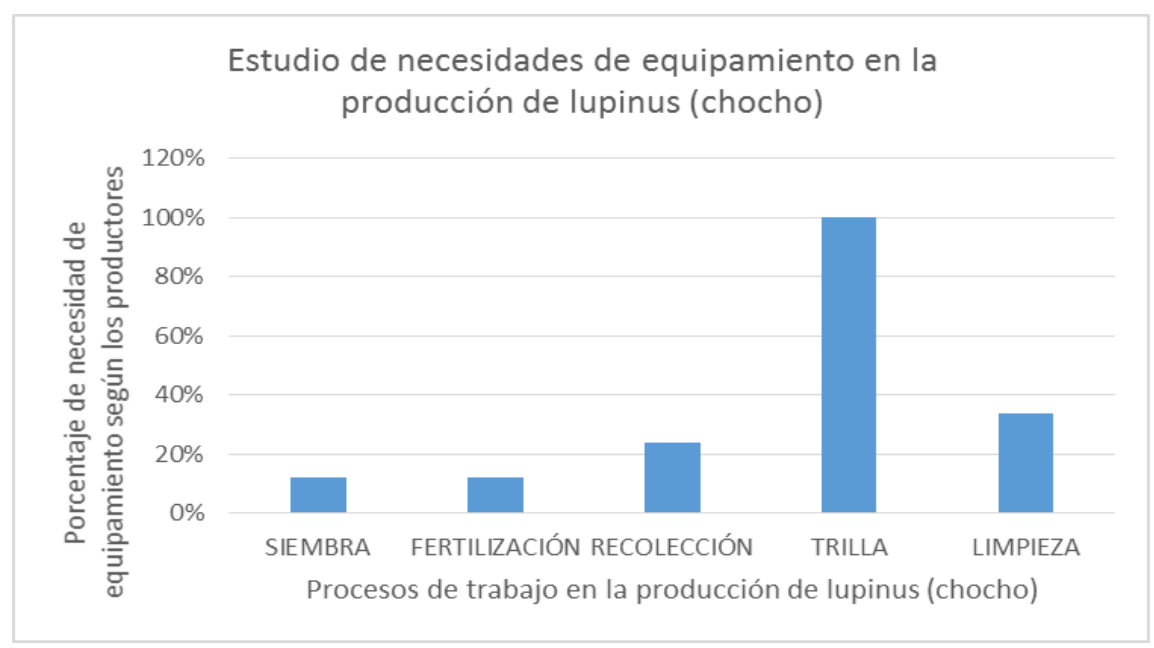

Figura 10. Necesidades de equipamiento en el proceso de producción de lupinus. Fuente: elaboración propia.

Las necesidades en cuanto a equipamiento en este producto están relacionadas al proceso de trilla y limpieza. El prototipo desarrollado para este efecto realizó el proceso de trilla y 
limpieza disminuyendo el tiempo de trabajo en una proporción de veinte veces a una respecto al trabajo manual.

La figura 11 expone las necesidades de equipamiento para la producción de fréjol.

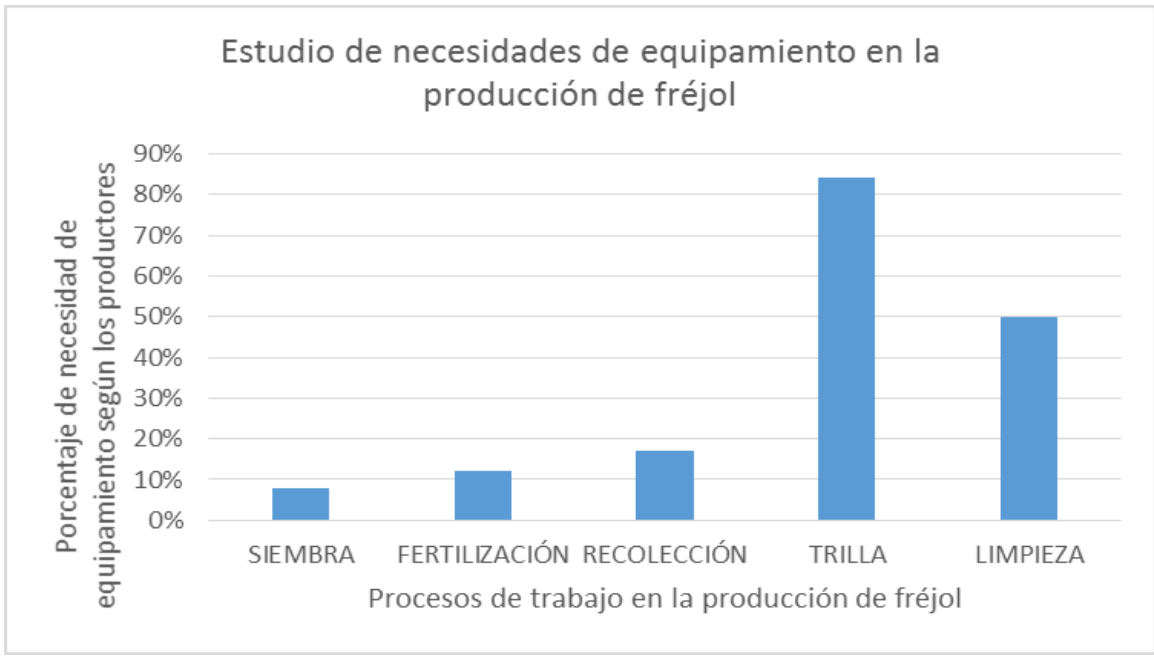

Figura 11. Necesidades de equipamiento en el proceso de producción de fréjol. Fuente: elaboración propia.

Al igual que el lupinus, el mayor requerimiento es en cuanto al proceso de trilla y limpieza.

En cuanto al nivel de formación, los productores, de un total de 712.037 pequeños y medianos productores (Sinagap, 2016), el 89,44\% de los mismos tiene formación primaria o no tiene formación, siendo el porcentaje de aquellos que no tienen formación alguna un $24,1 \%$. Esta información es fundamental en el proyecto ya que de este aspecto depende la propuesta de diseños a desarrollar y la forma de introducción de los equipos en este mercado.

La tabla 1 muestra las necesidades principales de desarrollo mencionadas por los agricultores para el equipamiento, con su nivel de importancia. El rango de importancia está entre 0 y 5 , siendo 0 de menor importancia y 5 de mayor importancia.

Tabla 1. Requerimientos para el desarrollo del equipamiento.

\begin{tabular}{|l|l|c|}
\hline No. & REQUERIMIENTOS QUE DEBE TENER EL EQUIPAMIENTO & IMPORTANCIA \\
\hline $\mathbf{1}$ & Fácil de operar & 4 \\
\hline $\mathbf{2}$ & Transportable & 3 \\
\hline $\mathbf{3}$ & Eficiente y eficaz & 4 \\
\hline $\mathbf{4}$ & Bajo costo & 5 \\
\hline $\mathbf{5}$ & De fácil mantenimiento & 4 \\
\hline $\mathbf{6}$ & Que tenga servicio técnico & 3 \\
\hline $\mathbf{7}$ & Segura al operar & 4 \\
\hline $\mathbf{8}$ & Amigable con el ambiente & 2 \\
\hline
\end{tabular}

Fuente: elaboración propia. 
En base a estos requerimientos se desarrollaron una serie de prototipos que se muestran a continuación.

La figura 12 muestra el equipo de deshojado diseñado específicamente para la variedad seco suave, que tiene una capacidad promedio de 100 mazorcas por minuto; manualmente, el productor realiza esta operación con un promedio de 12 mazorcas por minuto.

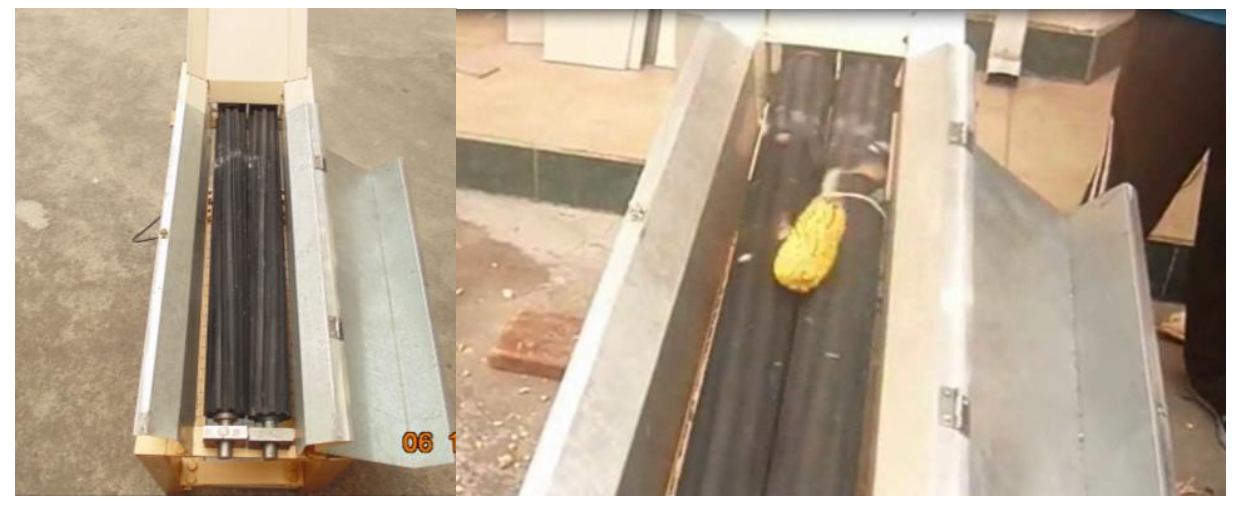

Figura 12. Equipo deshojador de maíz.

Fuente: autores (2014).

La figura 13 muestra 2 prototipos clasificadores de granos, el de mayor capacidad clasifica cuatro tamaños de producto a razón de $900 \mathrm{~kg}$ por hora. El trabajo en forma manual, en el caso de maíz, se realiza a razón de $40 \mathrm{Kg}$ por hora con una persona.

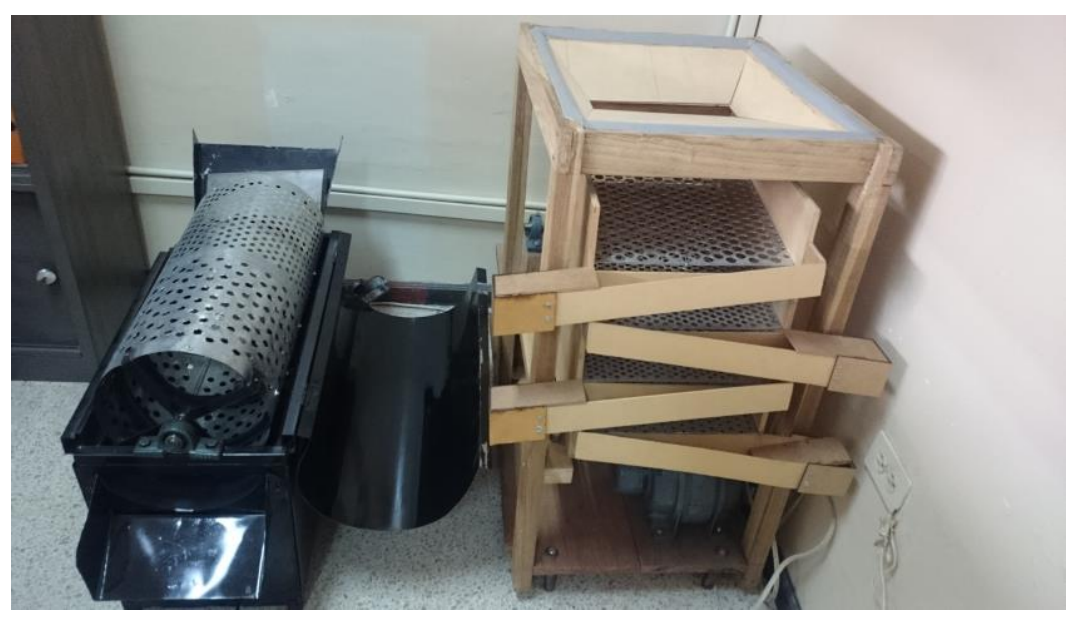

Figura 13. Prototipos clasificadores de granos.

Fuente: autores (2016).

La figura 14 muestra un prototipo de equipo desgranador de maíz. 


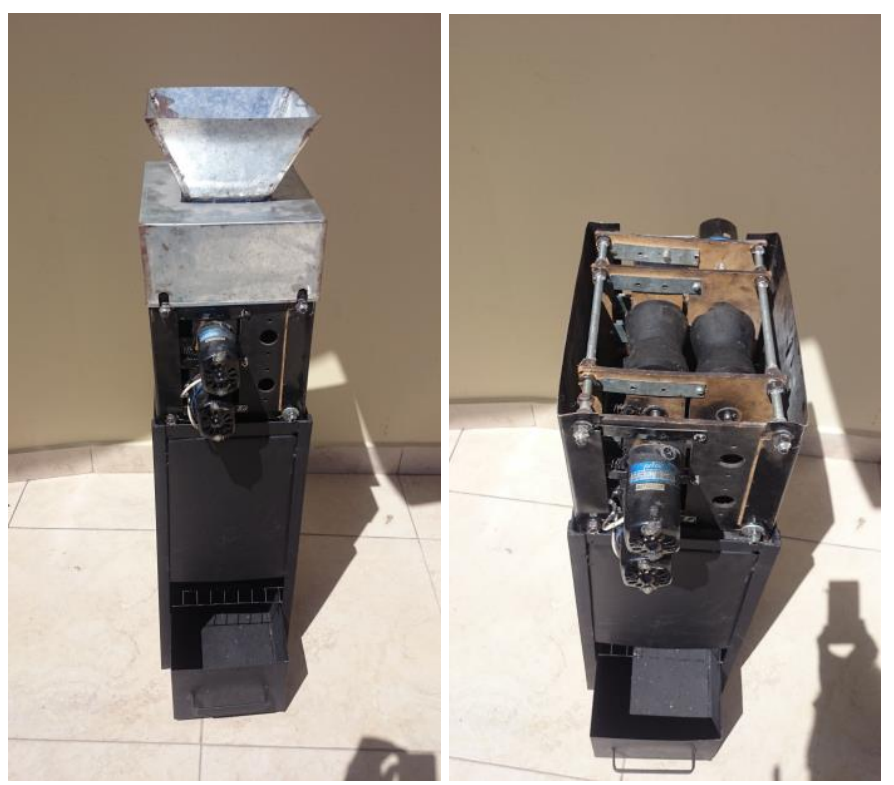

Figura 14. Prototipo desgranador de maíz.

Fuente: autores (2017).

El equipo desgrana 75 mazorcas por minuto. De forma manual el promedio de esta operación es de 3 mazorcas por minuto.

La figura 15 muestra un mecanismo de siembra de presición monograno colocado sobre un robot tetrápodo. De este sistema se han obtenido los primeros resultados y se encuentra en un proceso de mejora.

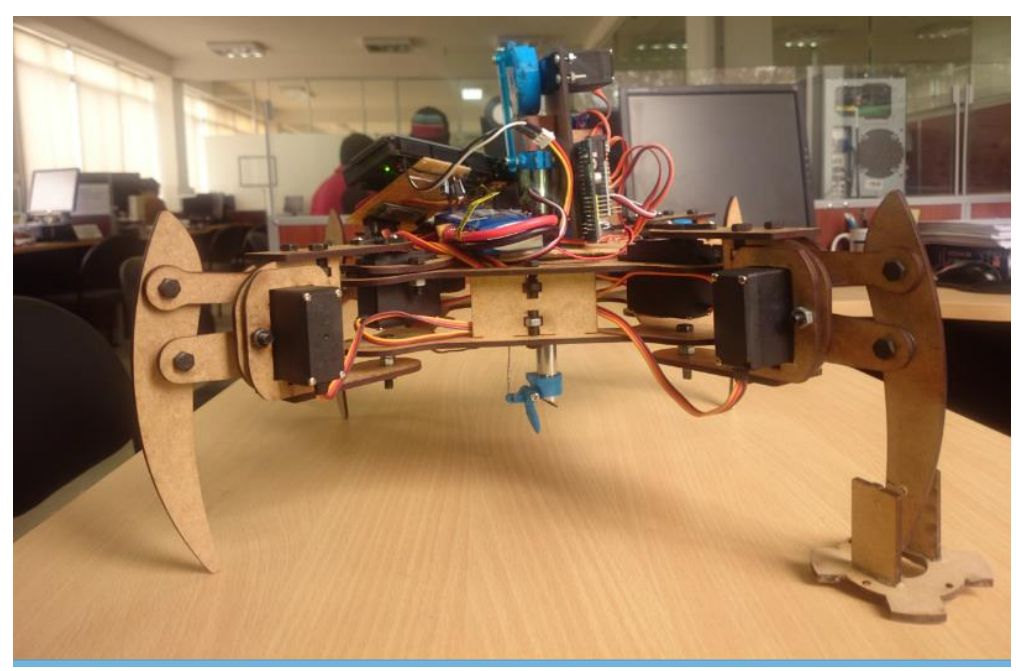

Figura 15. Prototipo de robot tetrápodo sembrador con mecanismo de siembra monograno Fuente: El autor (2016).

Todo este equipamiento se desarrolló de manera exitosa en base a las necesidades del productor andino y tiene las características de eficiencia, efectividad y bajo costo y constituyen una alternativa de solución a los problemas de los agricultores. 


\section{CONCLUSIONES}

- El producto que requiere mayor atención en cuanto al desarrollo de equipamiento en sus distintas etapas de producción es el maíz.

- En las variedades de productos estudiados, el proceso de cosecha es el que presenta un mayor requerimiento de equipamiento, lo cual se debe a la laboriosidad de esta etapa de trabajo.

- Los prototipos desarrollados probaron la factibilidad de desarrollo de equipamiento apropiado para los productos andinos en estudio y la efectividad de los mismos respecto a procesos manuales de trabajo.

- La totalidad de productores han mostrado interés en el desarrollo y posible adquisición del equipamiento.

\section{RECOMENDACIONES}

- Los procesos de siembra y fertilización, según los productores, no demandan mayor equipamiento. Esta percepción es debida a la falta de conocimiento de la forma correcta de producción y solamente analizan la problemática desde el punto de vista del esfuerzo que requieren las actividades, pero no considera la mayor productividad que podría tener al realizar los otros procesos de manera tecnificada. Por ello, la introducción de soluciones técnicas en estas etapas de producción tendría una estrategia diferente y debería enfocarse en demostrar resultados para tener aceptación.

- Debido al nivel de ingresos de los productores, el equipamiento a desarrollar debe ser de bajo costo.

- Las entidades gubernamentales, encargadas de realizar la difusión y capacitación en buenas prácticas en los procesos productivos agrícolas, disponen de presupuestos para la compra y difusión de maquinaria con el sector campesino. Por ello, una estrategia de comercialización del equipamiento desarrollado sería la de trabajar en conjunto con la entidad y proveerla para que sea ella la que se encargue de la entrega a los campesinos. Esto ayudaría a los mismos a obtener créditos gubernamentales o subsidios para la adquisición. 


\section{REFERENCIAS BIBLIOGRÁFICAS}

Albornoz, I. (2010). La inserción internacional de la industria argentina de Maquinaria Agrícola. Posicionamiento económico estratégico en el marco de las cadena globales de Valor y perspectivas futuras.

CDEI-UPC. (Febrero de 2012). http://www.cdei.upc.edu/es/noticia/15.

CEPAL, FAO, IICA. (2013). Perspectivas de la agricultura y el desarrollo rural en las Américas. San José.

CEPAL, FAO, IICA. (2014). Perspectivas de la agricultura y del desarrollo rural en las américas: una mirada hacia América Latina y el Caribe 2014. San José .

CEPAL, FAO, IICA. (2015). Perspectivas de la agricultura y del desarrollo rural en las Américas: una mirada hacia América Latina y el Caribe 2015-2016. San José.

De los Ríos, J. (2008). La agricultura latinoamericana y sus posibilidades de adapatación a los cambios climáticos globales. IDeAS, 2(1), 53-75.

Gleason, M. (2006). Maquinaria agrícola. Revista de geografía agrícola, 129 - 154.

Griffin, A. y Hauser, J. (1993). The voice of the customer. Marketing Science, 12(1), 1-27.

Kienzle, J., Ashburner, J. y Sims, B. (2013). Mechanization for Rural Development. Roma: FAO.

Martinez, L. (2005). Migración internacional y mercado de trabajo rural en el Ecuador. Quito: Flacso.

Pérez, P. F. (1991). Las Reformas Agrarias y la modernización económica. Scripta Vetera.

Riba, C. (2002). Diseño concurrente. Barcelona: UPC.

Sabourin, E., Samper, M., Le Coq, J., Massardier, G., Sotomayor, O. (2014). El surgimiento de políticas públicas para la agricultura familiar en América Latina: Trayectorias, tendencias y perspectivas. Cadernos de Ciência \& Tecnologia, 31(2), 189-226.

Sinagap. (2016). TABLA 1. NUMERO DE UPAS Y PERSONAS PRODUCTORAS POR TAMAÑOS DE UPA,SEGÚN PRINCIPALES CARACTERISTICAS . Quito: Sinagap.

Tapia, M., Fries, A. (2007). Guía de campo de los cultivos andinos. Lima: FAO y ANPE. 